Musées, Patrimoine et Culture scientifiques et techniques

\title{
Expériences de visite : de la transmission à la liction
}

Daniel Schmitt et Muriel Meyer-Chemenska

\section{OpenEdition \\ Journals}

Édition électronique

URL : http://journals.openedition.org/ocim/1440

DOI : $10.4000 /$ ocim. 1440

ISSN : 2108-646X

Éditeur

OCIM

Édition imprimée

Date de publication : 1 septembre 2014

Pagination : 17-23

ISSN : 0994-1908

Référence électronique

Daniel Schmitt et Muriel Meyer-Chemenska, "Expériences de visite : de la transmission à la liction», La Lettre de l'OCIM [En ligne], 155 | 2014, mis en ligne le 01 septembre 2016, consulté le 19 avril 2019 URL : http://journals.openedition.org/ocim/1440 ; DOI : 10.4000/ocim.1440

Ce document a été généré automatiquement le 19 avril 2019

Tous droits réservés 


\title{
Expériences de visite : de la transmission à la liction
}

\author{
Daniel Schmitt et Muriel Meyer-Chemenska
}

\section{Les expériences des visiteurs}

1 Pouvons-nous avoir accès à l'expérience vécue par les visiteurs lorsqu'ils effectuent un parcours de visite? Comment construisent-ils du sens en situation naturelle, sans médiateur, sans tiers, avec tous les dispositifs que nous avons conçus pour eux? En tant que muséographes et médiateurs, nous parlons souvent en leur nom pour justifier des choix de conception, mais nous savons bien que les visiteurs sont avant tout des êtres doués d'une raison autonome et d'une trajectoire de vie singulière. C'est en ce sens que Daniel Schmitt a réalisé un travail de recherche sur l'expérience des visiteurs en situation de visite naturelle dans le musée de L'CEuvre Notre-Dame et au musée zoologique de Strasbourg et où il a développé un processus d'enquête sous la forme d'entretiens en resitu subjectif ou entretien RSS (voir « Les entretiens en re-situ subjectif RSS »). 


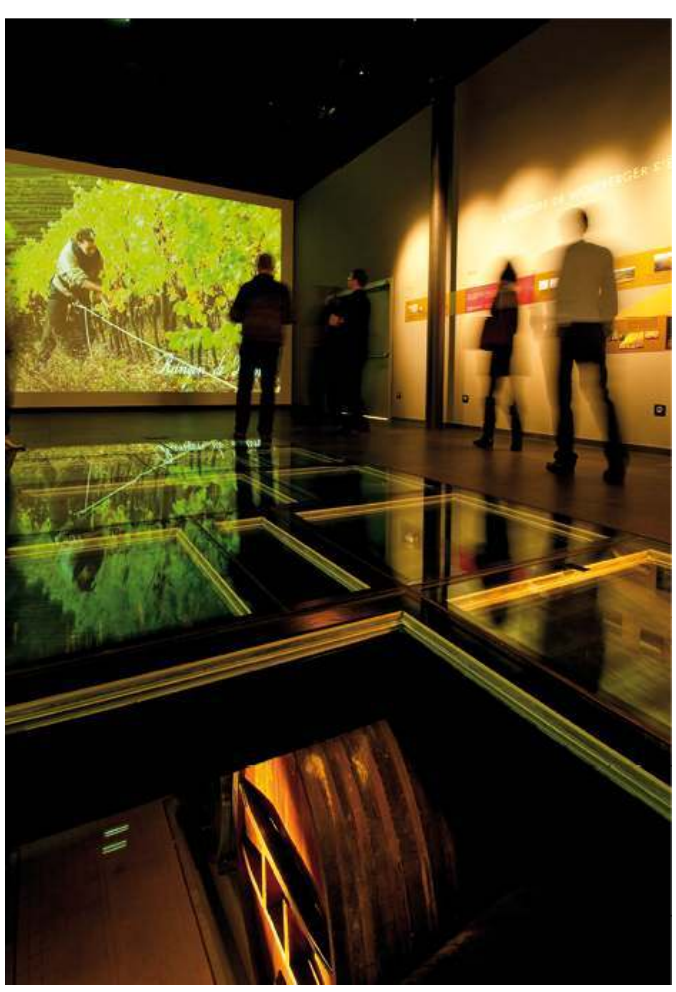

(c) Daniel Schmitt

Les entretiens RSS confirment d'abord que la simple observation des visiteurs in situ durant leur parcours ne permet pas d'interpréter leur expérience ou de tirer des conclusions sur la nature et les modalités de leurs apprentissages. Cette forme d'éthologie décrit avant tout le déplacement relatif du corps du visiteur dans l'espace, mais ne permet pas d'établir des corrélations entre un comportement et le domaine cognitif des visiteurs, c'est-à-dire ce qu'ils pensent, imaginent, ressentent, attendent... D'un point de vue scientifique, l'observation du visiteur s'arrêtant devant une sculpture est une description du chercheur qui séquence un flux continu d'activité en fonction de sa propre perception et construit le "parcours" du visiteur. Cela ne nous apprend strictement rien quant à l'expérience vécue par le visiteur tant que cette expérience n'est pas verbalisée par le visiteur lui-même. Du point de vue des visiteurs pendant la visite, il n'y a pas de parcours, mais des concaténations d'expériences. Par exemple dans un musée d'histoire de l'Art médiéval, si Olivier s'arrête et fixe une sculpture, est-ce parce qu'il la trouve particulièrement intéressante? Tout au contraire, Olivier s'arrête précisément devant cette statue parce qu'elle ne lui paraît pas remarquable et qu'elle lui permet de "faire une petite pause" :

"Alors en fait je fais plutôt le vide... Je fais le vide devant notamment les sculptures qui... devant laquelle je ne vais pas fixer intensément quelque chose... là ce petit Sacrifice d'Abraham pff... reste relativement classique donc c'est peut-être un moment où hop! mon esprit va faire une petite pause... ça je le fais inconsciemment, là je vois que mon esprit s'est fixé sur quelque chose qui n'est pas terrible... ce qui me permet d'être beaucoup plus... réceptif notamment quand je lis un cartel". 
4 À l'inverse, passer en trombe dans un espace du parcours ne signifie pas que le visiteur n'y connaît rien ou n'est pas sensible aux messages. Daniel, un ancien barman, traverse sans s'arrêter la salle du Jubé qui présente la statuaire médiévale provenant de la cathédrale de Strasbourg du même musée. Daniel dira au cours de l'entretien qu'il est aussi miniaturiste et qu'à ce titre, il sait dater "au premier coup d'œil" les sculptures du Moyen-Âge à la facture de leur drapé :

5 "Donc voilà je rentre dans une salle... sur le Moyen-Âge, qui n'est pas le but de ma visite de ce musée puisque je recherche tout ce qui a trait à la Renaissance, toutes les sculptures de la Renaissance... tout ce qui a trait donc à cette époque-là, 1500 jusqu'à 1525 exactement... au premier coup d'œil je vois que c'est antérieur à 1450 donc c'est plein Moyen-Âge donc ce n'est pas la période qui m'intéresse...".

6 Les entretiens RSS rendent compte en second lieu de l'étonnante richesse des expériences des visiteurs. Notre environnement tel que nous le percevons dépend fondamentalement de notre structure biologique, de notre

7 histoire et de nos attentes. Nous n'avons pas la possibilité d'accéder à notre environnement tel qu'il est en lui-même, c'est pourquoi Didier Bottineau (2011) nomme ce monde inconnaissable le X-monde. En revanche nous connaissons avec certitude la relation que nous entretenons avec ce X-monde, c'est-à-dire notre monde propre, et c'est ce monde propre qui surgit dans les entretiens avec les visiteurs.

8 Par exemple, au musée zoologique de Strasbourg, le diorama Arctique présente un morse parmi d'autres espèces de cet écosystème. Chaque visiteur aborde ce dispositif d'une manière différente. L'un, Mohan s'étonne de la taille de l'animal :

9 " Là on se posait une question, si celui-là il est vrai ou faux... parce que je n'ai jamais vu un animal aussi grand de ma vie... un animal comme ça aussi géant, parce que quand on les voit à la télé, on les voit très petits... c'est vraiment... j'imagine plus une baleine aussi grande ou une moitié de baleine mais pas un morse... le morse là il est énorme... juste à côté de lui on est des nains quoi...".

10 D'autres visiteurs tentent d'établir du sens en identifiant et en reliant différents éléments. Lisa par exemple identifie des traits de ressemblance, Aurore circonscrit des traces sur la peau, Axel remarque les dents du morse et il pense alors à l'éléphant de mer qui est exposé plus loin :

11 - Lisa (12 ans) : "J'ai essayé de regarder s'ils se ressemblaient un petit peu pour savoir s'ils étaient un peu de la même famille...".

12 - Aurore (23 ans) : " C'est un morse [...] je trouve que sa peau est vieille... oui je crois que ce qui m'a marquée c'était les entailles dans la peau... c'est que ça fait longtemps qu'ils sont là ces animaux quoi... c'est pas des animaux qui viennent d'être empaillés ou qui viennent d'être faits... c'est vraiment vieux...".

13 - Axel (6 ans) : "C'est l'éléphant de mer... je trouve qu'il est assez rigolo... mais c'est parce qu'on dirait que c'est un énorme morse sans dents, donc c'est quand même assez rigolo... oui c'est assez particulier, on dirait que c'est un morse sans longues dents...".

14 Les "entailles" sur la peau perçues par Aurore constituent un fait qui s'appuie sur le réel, mais qui a lieu dans le monde propre d'Aurore. Ces marques ne sont pas perçues comme telles par les autres visiteurs, elles n'existent tout simplement pas dans leur monde. Notons que la réalité physique des expôts dans le X-monde est la même pour tous les visiteurs. Mais c'est bien l'expérience singulière des visiteurs - notamment au cours de 
négociations interpersonnelles - qui construit une réalité stable et le monde propre de chaque visiteur.

\section{Que signifie "comprendre" du point de vue des visiteurs?}

Il existe une infinité de façons de circonscrire des éléments du réel, de les relier entre eux afin de construire du sens. On peut comparer le regard de deux statues pour savoir si elles ont été réalisées par le même sculpteur. On peut regarder avec empathie un animal naturalisé pour ressentir son vécu. On peut accrocher des courroies à des poulies pour faire tourner un ensemble d'éléments dans un centre de culture scientifique.

Quelle que soit l'activité des visiteurs, à chaque fois qu'une personne affirme qu'elle a compris, nous devons entendre qu'elle a réussi à résoudre l'intrigue qu'elle a elle-même mise en place - avec ou sans l'aide des concepteurs. Cette personne a isolé un ou plusieurs éléments qui ont fait émerger des attentes et elle mobilise des savoirs afin de résoudre cette intrigue. Lorsqu'à partir de cet ensemble hétérogène d'objets, de questions et de savoirs, le visiteur établit une relation qui paraît lui convenir, cette relation est condensée par la verbalisation "j'ai compris". Il s'agit d'une réponse qui convient à la situation telle que la perçoit le visiteur: il ou elle "comprend" ce qu'il y avait lieu de comprendre, même si cela peut être très éloigné des intentions des concepteurs.

17 La résolution des intrigues recouvre des modalités très variées: on peut circonscrire, distinguer, comparer, assembler, dissocier, relier des objets, des indices, des formes, des idées, des couleurs, des sensations. On peut aussi se souvenir, avoir peur, être impressionné... Mais quelle que soit la résolution, à chaque fois qu'un visiteur trouve une réponse qui convient à la question qu'il se pose dans un contexte tel qu'il le perçoit, il construit du sens, il crée, renforce ou diminue une connaissance, tandis qu'une sensation de plaisir accompagne le décours de cette résolution (Schmitt, 2014). Voici trois séquences où les visiteurs construisent ou renforcent des connaissances :

18 - Genny : "Moi ce qui m'intéressait aussi c'est ça... des êtres en suspension comme au cinoche dans les films de danse où souvent tu vois des gens qui sont épinglés, qui descendent dans des flous artistiques magnifiques... ça aussi j'ai beaucoup aimé, tu vois ces attitudes d'appui... j'ai trouvé ça tellement contemporain dans la représentation du corps, c'est tellement fou et tellement libre dans la gestuelle...".

19 - Gwenn : "J'aime vraiment bien la présentation des femmes, des Vierges... les femmes n'étaient pas bien représentées donc la seule manière... enfin d'être une femme correcte c'était... oui être pieux, être vierge... suivre les directives de l'Église et si jamais on ne faisait pas ça, on était soit une sorcière, soit folle... j'aime bien ce petit fossé entre la sainte femme et la femme considérée comme la sorcière, l'enfer enfin j'aime bien... j'aime bien les deux côtés...".

20 - Fanny : "Je lui demande [à mon père] les instruments de musique parce que mon père a fait des études au conservatoire donc... il essaye d'identifier chaque instrument... sur les trois statues de ce côté et sur les trois autres... les noms des instruments... je trouve que c'est une salle impressionnante à cause du côté massif des pièces exposées et aussi parce qu'on voit des détails qu'on ne voit pas sur l'ensemble de la cathédrale...". 
21 La diversité des connaissances émerge du découpage de l'environnement associé à des attentes et des savoirs singuliers. À ce stade des recherches sur le cours d'expérience des visiteurs dans les musées, la liberté de découper le monde en éléments puis de les relier selon ses envies, ses attentes et ses savoirs semble jouer un rôle essentiel quant à la capacité à construire du sens dans un parcours de visite.

Les connaissances réellement construites par les visiteurs. Genny : "des êtres en suspension comme au cinoche dans les films de danse...". Gwenn : "j'aime vraiment bien la présentation des femmes, des Vierges". Fanny : "je trouve que c'est une salle impressionnante..." (captures d'écran extraits des perspectives subjectives en rapport avec les verbalisations des visiteurs).
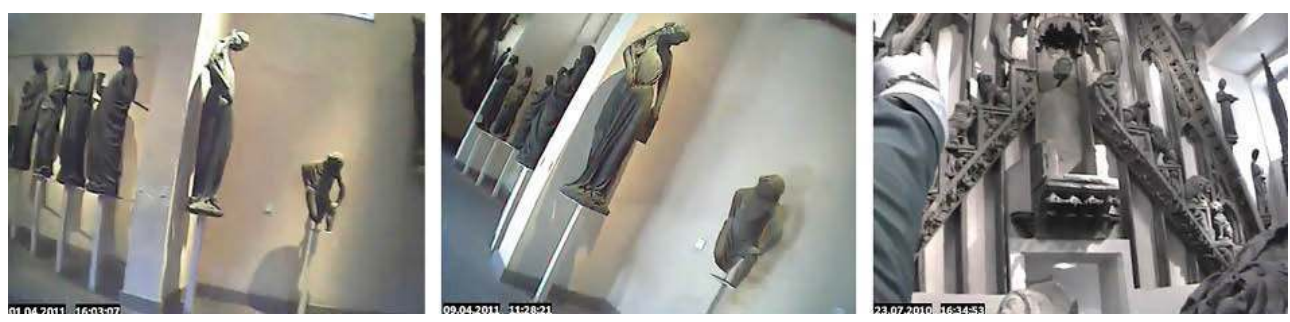

(C) D. Schmitt

\section{Du particulier au général : typicaliser les attentes et les savoirs}

22 Le "cours d'expérience" est défini ici en référence à Jacques Theureau (2006) comme ce qui fait sens pour un visiteur dans le cours de sa visite, au fur et à mesure de son activité. Les cours d'expérience des visiteurs dans un parcours muséal, analysés à partir d'entretiens en re-situ subjectif nous permettent de recenser les attentes des visiteurs à chaque instant de leur visite. En typicalisant ces attentes, nous faisons apparaître des classes thématiques d'anticipations. Elles révèlent à la fois ce que les visiteurs espèrent trouver et ce qu'ils ne trouvent pas. Par exemple dans le musée de l'ÆEuvre Notre-Dame à Strasbourg qui propose une découverte de sept siècles d'art dans la région du Rhin supérieur, Roman, Pierce et Alain se trouvent dans la salle du Jubé qui présente la statuaire de la cathédrale. L'un, Roman s'attarde devant un schéma de construction de la cathédrale, tandis que Pierce et Alain regardent des sculptures :

- Roman : "Je me pose des questions, comment ils ont fait pour obtenir ce résultat parce qu'aujourd'hui il y a des milliers de plans... je ne sais pas comment ils communiquaient entre eux, il me manque... ici j'aimerais bien par exemple voir... j'ai vu une fois des plans de la tour Eiffel et maintenant je me dis pourquoi pas de la cathédrale... et je n'ai pas vu... quelqu'un m'avait dit, pour bâtir une grande église, ils ont de deux, trois, quatre planches de dessin, ils font comme ça, mais j'ai essayé de retrouver [ça] ici... bon, j'ai pas trouvé...".

24 - Pierce : "Je la vois mal placée... je ne vois pas tellement son sens, on la voit à la portée des yeux, mais je la vois mal placée en l'air... j'ai vu qu'il y avait un scellement justement en plomb qui devait soutenir sur l'arrière... les autres on comprend comment ils pouvaient être disposés, ça je ne vois pas comment il pouvait être... oui j'aurais bien aimé savoir comment il était disposé... ça reste une énigme...".

25 - Alain: "J'aimerais qu'on essaye de me dire qu'est-ce que ça représente, ce que je suis censé voir en fait là. Là, je vois des statues, mais est-ce que ce sont des personnages importants? Est-ce que ce sont des personnages qui sont censés faire peur aux gens qui 
allaient à la messe à l'époque ? [...] il n'y a pas la mise en scène disant : bon les cathédrales à l'époque étaient des lieux où on essayait de terrifier la population... fallait pas faire de péchés etc. Il n'y a rien qui me dit tout ça dans cette salle-là".

Dans cet espace du musée, un tiers des visiteurs enquêtés aurait souhaité trouver des indications techniques : poids, fonction, méthode, temps de fabrication, organisation et logistique du travail, comparaison avec des éléments actuels ordinaires. Tandis qu'un autre tiers des visiteurs aurait aimé avoir plus d'indications sur les thèmes philosophiques et spirituels comme la perception du monde, les croyances au Moyen-Âge, le rapport aux personnages sculptés et aux animaux représentés. La typicalisation des attentes fait émerger des thématiques qui peuvent servir à enrichir la médiation proposée. Ici le thème de la technique de production des œuvres et le thème du contexte philosophique du Moyen-Âge peuvent aider les visiteurs à se lier aux œuvres.

De la même façon, en typicalisant les savoirs mobilisés par les visiteurs dans le cours de leur visite, nous pouvons identifier les références clés qui participent à la construction de sens. Dans le musée de l'CEuvre Notre-Dame, un tiers des visiteurs enquêtés mobilisait à un certain endroit du parcours une représentation négative du Moyen-Âge verbalisée par "mortifère, peur, terrifier, péchés, méfiance, puissances aveugles, démoniaques, douleurs, mauvais esprits, brutalité, bien, mal". Cette expérience était considérée comme positive, mais faisait place à une certaine déception dans la salle suivante, la salle de la statuaire de la cathédrale. Nous avons mis en évidence que le contexte spatial influençait significativement la mobilisation d'un registre de savoir. Les visiteurs qui mobilisaient une représentation négative du Moyen-Âge étaient particulièrement sensibles à la couleur noire des murs d'une petite salle précédant la salle de la statuaire. Les représentations mobilisées renvoyaient à un Moyen-Âge obscur tandis que la salle de la statuaire, blanche et lumineuse créait une sorte de dissonance et d'incompréhension pour une proportion significative de visiteurs.

Les attentes des visiteurs suggèrent des nouvelles thématiques. De gauche à droite : Roman aimerait des informations sur l'organisation du travail au Moyen-Âge, Pierce aimerait comprendre la fixation de la sculpture sur l'édifice, Alain aimerait qu'on lui raconte le sens des sculptures (captures d'écran extraits des perspectives subjectives en rapport avec les verbalisations des visiteurs).
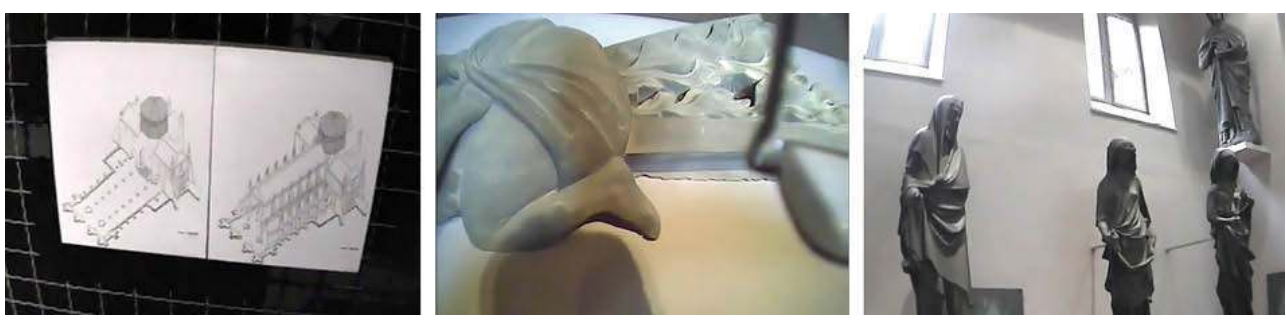

(c) D. Schmitt

\section{Comment nous incorporons la recherche dans notre pratique de muséographes}

Dans notre démarche de recherche et de praticien de la muséographie, nous avons confronté les intentions de médiation de l'institution dans le parcours de la statuaire avec les connaissances-type construites par les visiteurs (Schmitt, à paraître). Cette 
comparaison permet de mettre en exergue ce que l'on aimerait que les visiteurs "découvrent" et ce que les visiteurs construisent réellement.

Connaissances attendues et connaissances construites. Du point de vue de l'institution, les visiteurs devraient pouvoir percevoir les œuvres comme des originaux et des œuvres majeures, retrouver leur emplacement d'origine sur la cathédrale et remarquer les différences stylistiques dans les trois sections séparées par des arches.

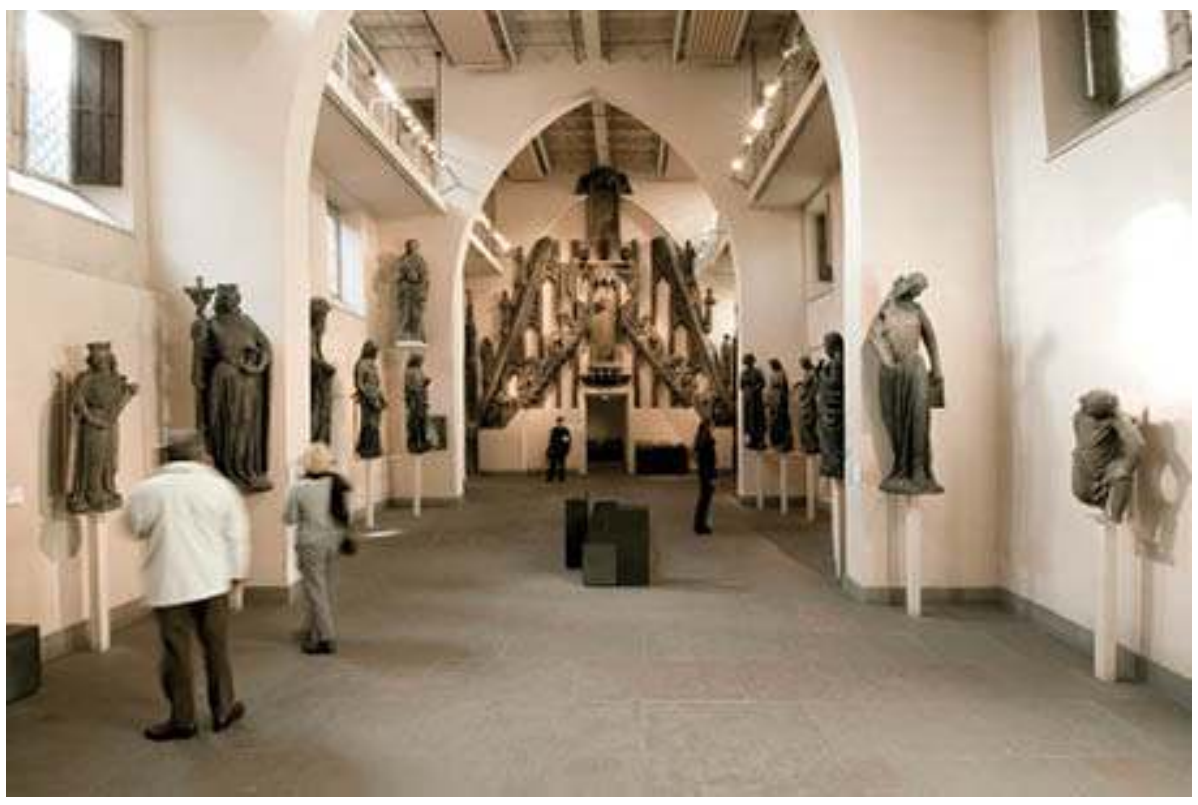

(c) D. Schmitt

Ainsi, nous avons demandé aux responsables du musée de faire le parcours de visite tout en décrivant ce que les visiteurs devraient selon eux réussir à comprendre. Il ressort de ces entretiens un certain nombre d'attentes de l'institution. Avant tout, les visiteurs doivent comprendre que les œuvres sont des originaux et des œuvres majeures. Ils doivent ensuite pouvoir repérer leur emplacement d'origine sur la cathédrale, comprendre les étapes de la construction de la cathédrale et les différentes écoles stylistiques. Puis nous avons comparé les connaissances typicalisées des visiteurs avec les connaissances-types anticipées par l'institution. Si la plupart des visiteurs enquêtés appréhendent les sculptures de la salle du Jubé comme des œuvres majeures, peu de visiteurs saisissent les différentes étapes de construction de la cathédrale, la place originelle des sculptures et les influences stylistiques des écoles de sculpture. Nous avons ainsi mis en évidence les écarts qui peuvent exister entre la perception par l'institution de la muséographie en place et celle qu'en ont les visiteurs.

Le musée a alors engagé des travaux de rénovation. Des dispositifs graphiques précisent et approfondissent certaines thématiques citées plus haut, un dispositif numérique de $\mathrm{QR}$ code permet d'accéder à un film documentaire sur la construction de la cathédrale, un dispositif tactile met en évidence les différentes étapes de la taille de la pierre. La petite salle d'introduction a été repeinte en blanc, ce qui n'invite plus à mobiliser des représentations négatives du Moyen-Âge.

31 En tant que concepteur d'exposition ou en tant qu'institution, nous ne pouvons jamais anticiper avec certitude ce qui sera perçu et compris par les visiteurs. Les visiteurs lorsqu'ils franchissent le seuil du musée, savent qu'il y a quelque chose à comprendre ou à 
trouver. Dès qu'ils trouvent une réponse à la question qu'ils se posent, ils "comprennent" et ils éprouvent un sentiment de plaisir. C'est bien le plaisir de la visite qui retient toute notre attention comme moteur de découverte, de compréhension, de délectation.

C'est pourquoi, ce qui nous importe en tant que muséographes, c'est de donner aux visiteurs des possibilités de comprendre, au sens de se relier aux expôts d'une façon qui leur convient à eux. Notre point de vue de concepteurs privilégie les lictions, ces mises en relation de perceptions, d'attentes, de savoirs et d'agir (Leleu-Merviel et Useille, 2008) qui nous permettent de "faire corps avec le monde" selon la belle formule de Bernard Darras et Sarah Belkhamsa (2008). Même si l'intention de "message" est nécessaire pour fédérer le groupe concepteur autour d'une cohérence de la grammaire scénaristique, la muséographie et la médiation ne consistent pas en une transmission de messages et de savoirs de l'institution vers le visiteur. D’ailleurs le "message" compris par les visiteurs, ce qui est construction de sens, nous échappe : au sens strict, il n'y a pas de transmission mais plutôt une émergence d'information, une "in-formation" comme le dit Francisco Varela (1989), c'est-à-dire du sens formé par et à l'intérieur du visiteur en fonction de ses attentes et de son histoire.

Influence de l'environnement sur les savoirs mobilisés : selon que la salle est peinte en noir (1) ou en blanc (2) les savoirs mobilisés ne sont pas les mêmes.

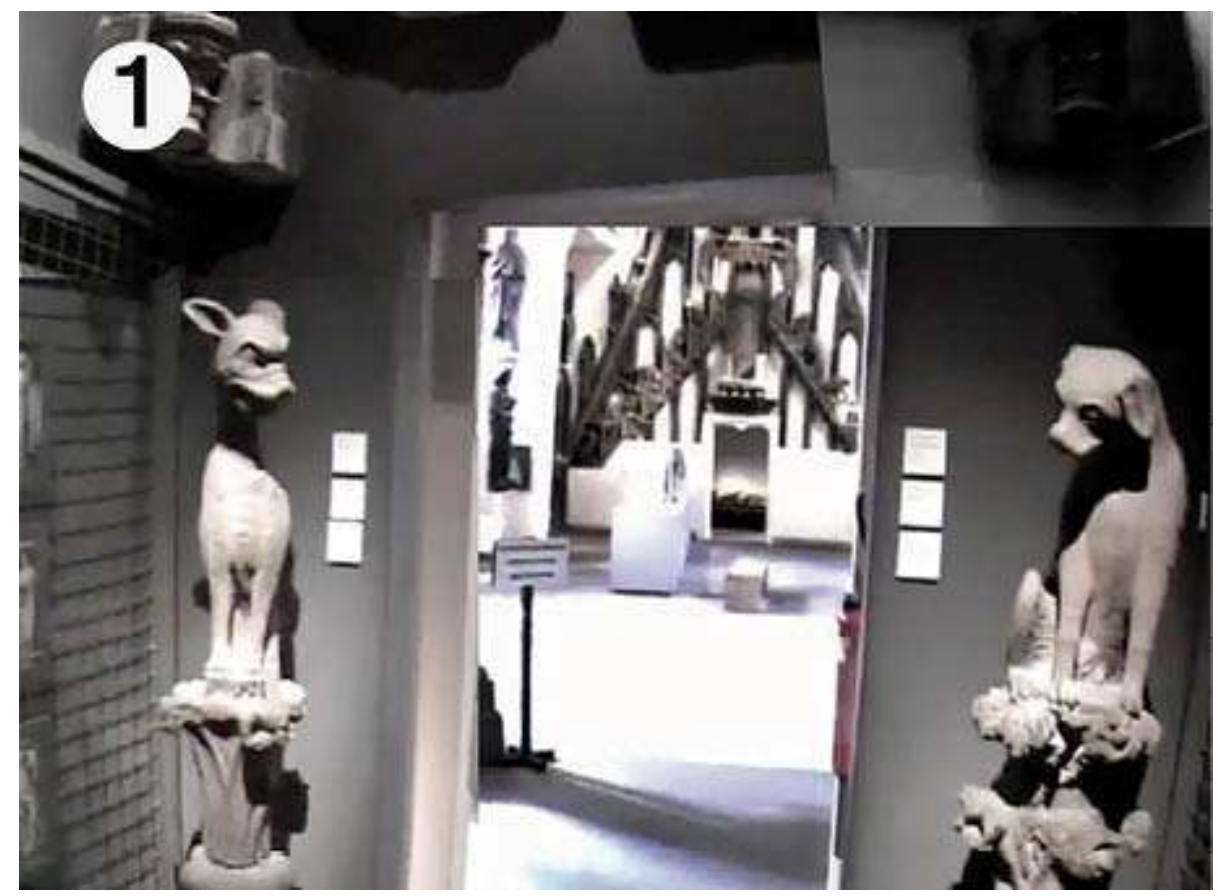




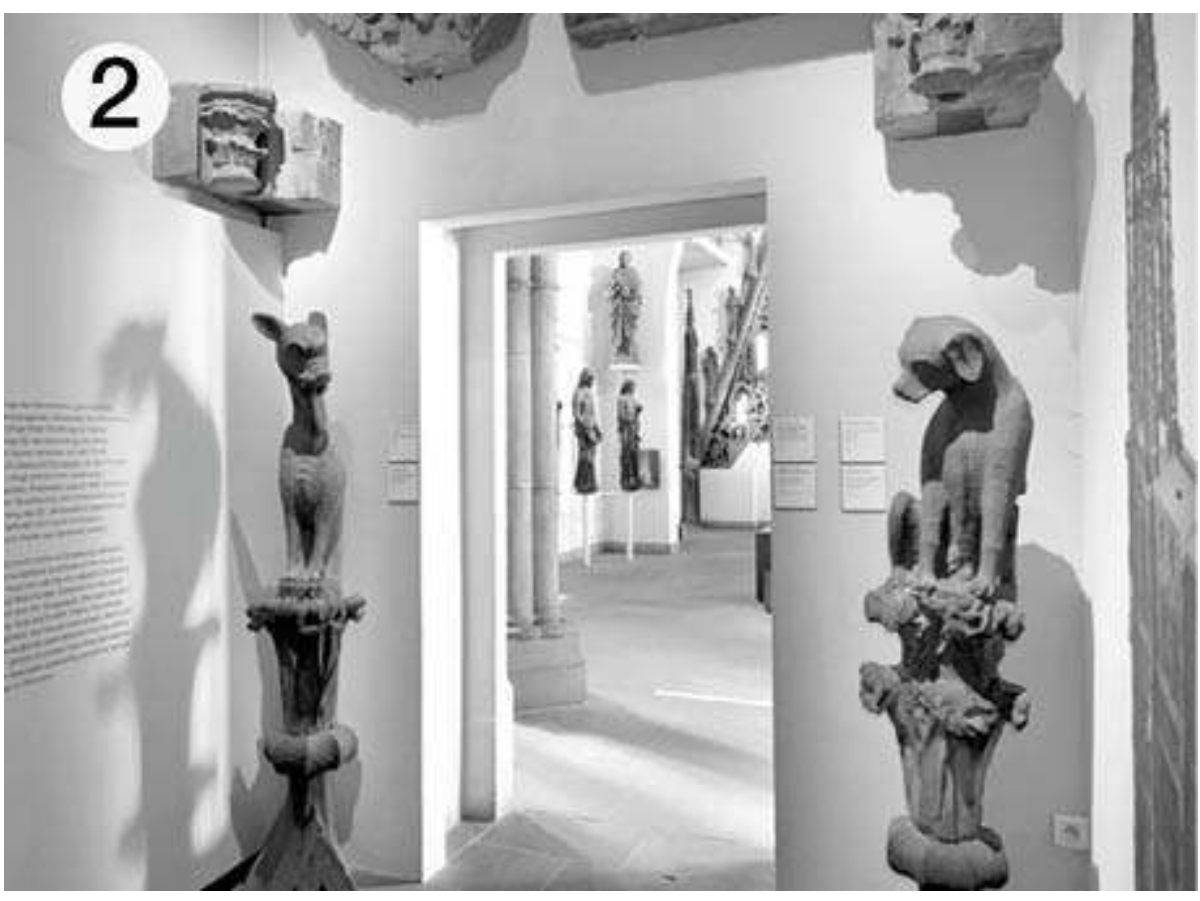

(C) D. Schmitt mais de créer des possibilités de lier le X-monde de l'exposition et de ses expôts aux mondes propres des personnes. Les expériences des visiteurs deviennent à la fois le moyen et la fin parce qu'il n'y a pas d'autre lieu où se construit la connaissance en situation naturelle. Les multiples relations visiteurs/expôts forment des motifs récurrents de liaison que l'on peut appeler des registres lictionnels. Ils sont aujourd'hui au cœur de notre réflexion sur la scénarisation des parcours. Car s'il est possible d'identifier les registres lictionnels, alors il devient possible de proposer des contextes qui favorisent des liaisons aux expôts.

L'approche par registre lictionnel en est à sa genèse, mais elle nous semble particulièrement prometteuse lorsque la muséographie s'appuie sur des dispositifs numériques (cartels numériques, visioguides, smartphones, $Q R$ codes, réalité augmentée...). Non seulement parce que ces dispositifs permettent aux visiteurs de disposer d'une grande variété de contenus scénarisés en relation avec leurs interactions. Mais surtout il devient possible de proposer aux visiteurs des contenus personnalisés à partir de leurs registres attentes-savoirs, identifiés dans le cours de leur activité.

ombreuses études explorent cette question de l'expérience des visiteurs. Jacqueline Eidelman, Hana Gottesdiener et Joëlle Le Marec (2013) en ont récemment rédigé une synthèse. Pour autant, la dimension "infra" de l'expérience, ces micro-instants où les visiteurs laissés à leur situation naturelle de visite perçoivent, échangent, découpent, mobilisent... en un mot et au sens le plus noble ces espaces-temps où les visiteurs "bricolent" le réel pour construire du sens, constitue un champ de recherche à venir. C'est dans cette perspective que notre démarche ne consiste pas à deviner ce qui conviendrait au visiteur, mais plutôt proposer des possibilités de liaisons en fonction de celles privilégiées et vécues par la personne au fur et à mesure de ses interactions dans l'espace et de ses expériences dans le parcours. 
'expérience muséale s'appuie sur des savoirs académiques négociés selon des formes étonnantes et ce qui importe pour les visiteurs, c'est davantage de se lier que de recevoir la parole de l'institution. Il s'agit de proposer des paroles de l'institution qui puissent s'inscrire dans les tensions attentes-savoirs récurrentes des visiteurs pour accompagner leurs résolutions en permettant une liaison aux collections selon leur histoire et leurs attentes. En d'autres termes, il s'agit de passer d'une muséographie de la transmission à une muséographie de la liction.

\section{Les entretiens en re-situ subjectif (RSS)}

37 Les entretiens réalisés en re-situ subjectif (Rix et Biache, 2004) ou entretiens RSS permettent de mobiliser la capacité de réminiscence des visiteurs et de leur faire verbaliser leur expérience de visite à l'issue de leur parcours tout en conservant la précision de l'expérience telle qu'elle a été vécue. La réminiscence est une forme de reviviscence produite par une excitation neurophysiologique. C'est en quelque sorte un effet "madeleine de Proust" provoqué et entretenu. Lorsque l'on stimule un visiteur avec la trace de son activité, par exemple en reproduisant les perceptions qu'il a eues lors de son parcours de visite, le visiteur ne revoit pas le film de son activité, mais il revit cette activité tout en disposant de temps pour décrire cette expérience.

Les entretiens réalisés en re-situ subjectif. Les visiteurs sont équipés d'une mini-caméra et laissés à leur parcours de visite (1). Les visiteurs sont ensuite invités à décrire leur expérience à partir de ce film tandis qu'une caméra placée derrière le visiteur enregistre l'image de la vidéo, l'entretien et les gestes du visiteur (2).
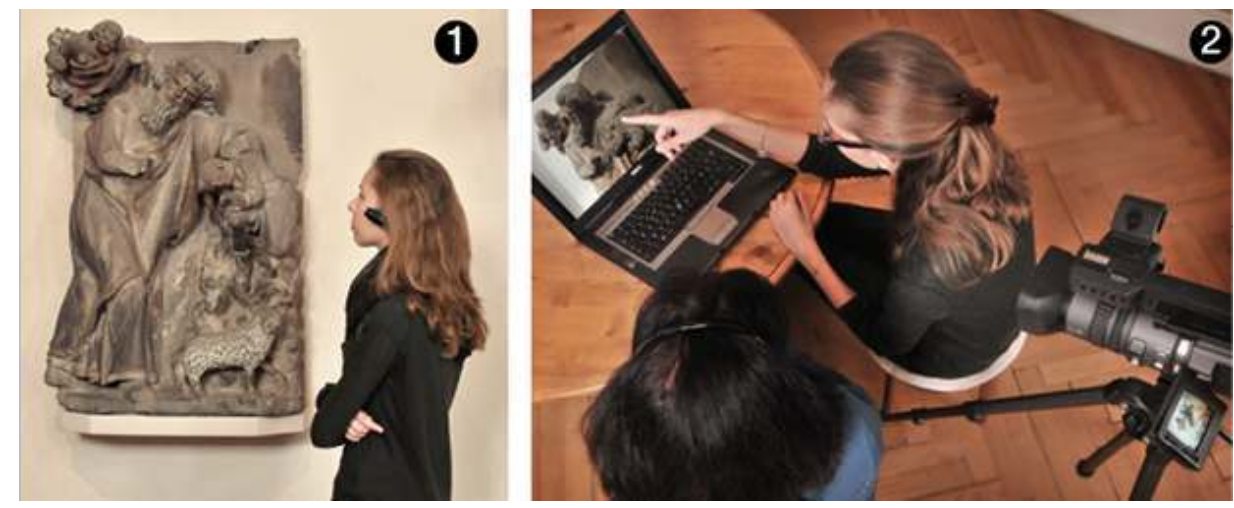

(c) D. Schmitt

Pour cela, nous équipons le visiteur d'une mini-caméra et d'un micro puis nous le laissons à son activité. Cette mini-caméra enregistre sa perspective visuelle et auditive. À l'issue de la visite, nous installons le visiteur devant un écran vidéo où nous diffusons l'enregistrement de sa propre perspective subjective et nous l'invitons à décrire et à commenter son expérience.

Le visiteur revit alors en qualité son expérience, découpe spontanément son activité en séquences qui sont signifiantes de son point de vue. Nous pouvons nous arrêter sur des séquences particulières pour laisser au visiteur le temps de décrire son activité, ses émotions, ses attentes... Une caméra placée derrière le visiteur et le chercheur enregistre l'entretien, les gestes du visiteur avec les images sur l'écran vidéo. Bien entendu ces verbalisations ne sont pas l'expérience elle-même, mais elles rendent compte de l'expérience des visiteurs avec précision, finesse et profondeur (Schmitt, 2012) parce 
qu'elles peuvent être considérées à la fois comme des descriptions symboliques acceptables du domaine cognitif du visiteur (Varela, 1989) et comme effet de surface du couplage du visiteur et de son environnement (Theureau, 2006). À l'issue de cet entretien, l'enregistrement obtenu est transcrit, les séquences vidéo et les verbalisations associées sont analysées avec le logiciel Advene (Aubert et al., 2012) : nous identifions ce qui est pris en compte par le visiteur, la nature de sa relation à l'environnement, ses attentes, les savoirs mobilisés, les connaissances construites et son état émotionnel.

Cette analyse nous permet de décrire le cours d'expérience, ce qui fait sens pour les visiteurs de leur point de vue, au fur et à mesure de leur activité de visite (Schmitt, 2013).

Analyse avec le logiciel Advene :

(1) les verbalisations visiteur-chercheur issues de l'entretien RSS sont transcrites verbatim et synchrones avec la vidéo subjective du parcours de visite.

(2) Les composantes du signe hexadique sont renseignées à partir de la vidéo subjective et des verbalisations visiteur-chercheur.

(3) Une vue hypervidéo est publiée : elle regroupe la vidéo subjective du visiteur commentée par visiteur et analysée par le chercheur.

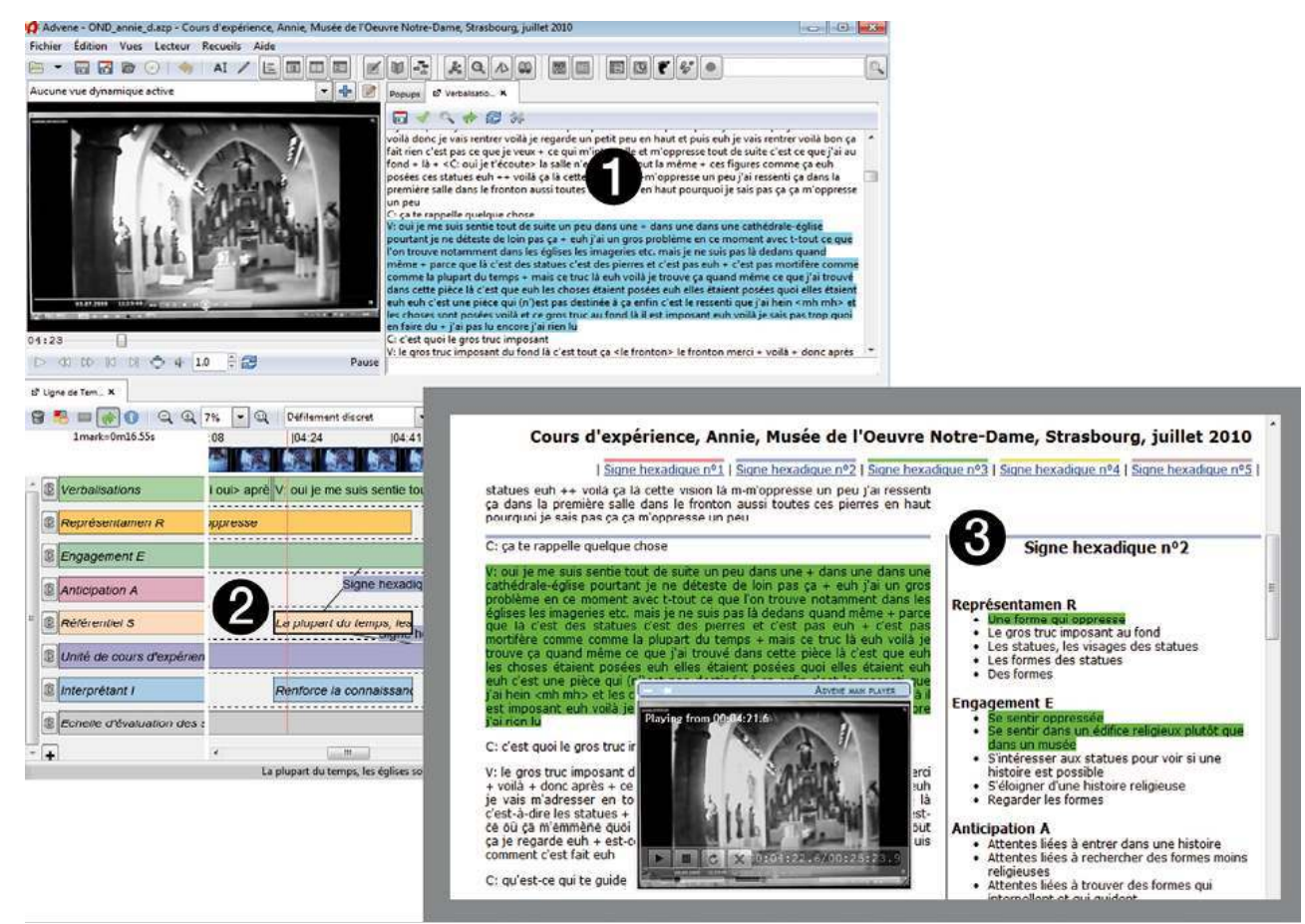

도. D. SCHMITT 


\section{BIBLIOGRAPHIE}

Aubert, O., Prié, Y. et Schmitt, D. Advene as a tailorable hypervideo authoring tool: a case study. Proceedings of the 2012 ACM Symposium on Document Engineering (DocEng), ACM New York, 2012, pp. 79-82.

Bottineau, D. Parole, corporéité, individu et société : l'embodiment entre le representationnalisme et la cognition incarnée, distribuée, biosémiotique et enactive dans les linguistiques cognitives, Intellectica, $n^{\circ} 56,2011$, pp. 187-220.

Darras, B. et Belkhamsa, S. Faire corps avec le monde. Étude comparée des concepts d'affordance, d'énaction et d'habitude d'action, Recherches en communication, ${ }^{\circ}$ 29, 2008.

Eidelman, J., Gottesdiener, H. et Le Marec, J. Visiter les musées : expérience, appropriation, participation, in Gottesdiener, H. et Davallon, J. (dir.) La muséologie : 20 ans de recherche, Culture et Musées, Hors Série, Éditions Actes Sud, 2013, pp. 73-113.

Leleu-Merviel, S. et Useille, P. Quelques révisions du concept d'information, in Papy, F. Problématiques émergentes dans les Sciences de l'information. Lavoisier/Hermès, collection Traité des Sciences et Techniques de l'Information, 2008, pp. 25-56.

Schmitt, D. Vers une remédiation muséale à partir de l'expérience située des visiteurs, Les Enjeux de l'information et de la communication (à paraître).

Schmitt, D. Ce que "comprendre" signifie pour les jeunes visiteurs dans un centre de culture scientifique, in Chavot P. et Masseran A.(dir.), Les cultures des sciences en Europe. 2. Dispositifs, publics, acteurs, institutions. Presses Universitaires de Nancy Lorraine. Coll. Questions de communication, 2014.

Schmitt, D. Décrire et comprendre l'expérience des visiteurs, ICOFOM Study Series - Le visiteur particulier : chacun et n'importe lequel d'entre nous. 2013, n 42 , pp. 205-216.

Schmitt, D. Expérience de visite et construction des connaissances : le cas des musées de sciences et des centres de culture scientifique. Thèse en sciences de l'information et de la communication, université de Strasbourg, 2012.

Rix, G. et Biache, M.-J. Enregistrement en perspective subjective située et entretien en re-situ subjectif : une méthodologie de la constitution de l'expérience, Intellectica, n 38, 2004, pp. 363-396.

Theureau, J. Le cours d'action : méthode développée. Toulouse : Octarès, 2006.

Varela, F. Autonomie et connaissance. Seuil, 1989.

\section{RÉSUMÉS}

Le programme de recherche "cours d'expérience" fondé sur des entretiens en re-situ subjectif vise à saisir et à comprendre comment les visiteurs des musées construisent du sens et des connaissances durant leur parcours de visite. Cette méthode permet de recenser les attentes et les savoirs mobilisés par les visiteurs, d'identifier les différentes façons de se relier au monde que 
constitue l'exposition et plus généralement d'envisager une nouvelle démarche de conception de la muséographie et de la médiation.

INDEX

Mots-clés : visite, musée, exposition

\section{AUTEURS}

\section{DANIEL SCHMITT}

maître de conférences à l'université de Valenciennes

daniel.schmitt@univ-valenciennes.fr

\section{MURIEL MEYER-CHEMENSKA}

directrice de l'agence de muséographie Métapraxis

muriel.meyer@metapraxis.fr 